\title{
On sumsets and spectral gaps
}

by

\section{Ernie Croot (Atlanta, GA) and Tomasz Schoen (Poznań)}

1. Introduction. Suppose that $S \subseteq \mathbb{F}_{p}$, where $p$ is a prime number. Let $\lambda_{1}, \ldots, \lambda_{p}$ be the absolute values of the Fourier coefficients of $S$ (to be made more precise below) arranged as follows:

$$
\widehat{S}(0)=\lambda_{1} \geq \lambda_{2} \geq \cdots \geq \lambda_{p}
$$

Then, as is well known, one can work out, as a function of $\varepsilon>0$ and a density $\theta=|S| / p$, an upper bound for the ratio $\lambda_{2} / \lambda_{1}$ which guarantees that $S+S$ covers at least $(1-\varepsilon) p$ residue classes modulo $p$. Put another way, if $S$ has a large spectral gap, then most elements of $\mathbb{F}_{p}$ have the same number of representations as a sum of two elements of $S$, thereby making $S+S$ large.

What we show in this paper is an extension of this fact, which holds for spectral gaps between other consecutive Fourier coefficients $\lambda_{k}, \lambda_{k+1}$, so long as $k$ is not too large; in particular, our theorem will work so long as

$$
1 \leq k \leq\lceil(\log p) / \log 2\rceil .
$$

Furthermore, we develop results for repeated sums $S+S+\cdots+S$.

It is worth noting that this phenomenon also holds in arbitrary abelian groups, as can be worked out by applying some results of Lev [4], [5], but we will not develop this here $\left(^{1}\right)$.

The property of $\mathbb{F}_{p}$ that we exploit is something we call a "unique differences" property, first identified by W. Feit, with first proofs and basic results found by Straus [7].

2000 Mathematics Subject Classification: Primary 11B50; Secondary 11B34, 05D99.

Key words and phrases: additive combinatorics, sumsets, pseudorandom functions, spectral gaps.

$\left({ }^{1}\right)$ In some of these general groups, the results are rather poor compared with the $\mathbb{F}_{p}$ case. For example, they are poor in the case where one fixes $p$ and works with the additive group $\mathbb{F}_{p}^{n}$, where one lets $n \rightarrow \infty$. The reason is that if one fixes a large subgroup of this group, and then lets $f$ be its indicator function, then $f$ will have a large spectral gap, and yet $\operatorname{supp}(f * f)$ will equal that $\operatorname{subgroup,~meaning~} \operatorname{supp}(f * f)$ cannot be a $1-\varepsilon$ fraction of the whole group. 
Before we state the main theorems of our paper, we will need to fix some notation. First, for a function $f: \mathbb{F}_{p} \rightarrow \mathbb{C}$, we define its normalized Fourier transform as

$$
\widehat{f}: a \mapsto \mathbb{E}_{z}\left(f(z) e^{2 \pi i a z / p}\right),
$$

where $\mathbb{E}$ here denotes the expectation operator, which in this context is defined for a function $h: \mathbb{F}_{p} \rightarrow \mathbb{C}$ as

$$
\mathbb{E}_{z} h(z):=p^{-1} \sum_{z \in \mathbb{F}_{p}} h(z) .
$$

If the function $h$ depends on $r$ variables, say $z_{1}, \ldots, z_{r}$, we define

$$
\mathbb{E}_{z_{1}, \ldots, z_{r}} h\left(z_{1}, \ldots, z_{r}\right):=p^{-r} \sum_{z_{1}, \ldots, z_{r} \in \mathbb{F}_{p}} h\left(z_{1}, \ldots, z_{r}\right) .
$$

We will then let $\lambda_{k}$ denote the $k$ th largest absolute value of a Fourier coefficient of $f$; in other words, we may write $\mathbb{F}_{p}:=\left\{a_{1}, \ldots, a_{p}\right\}$, where upon letting $\lambda_{i}:=\left|\widehat{f}\left(a_{i}\right)\right|$, we have

$$
\lambda_{1} \geq \cdots \geq \lambda_{p}
$$

We define the convolution of $r$ functions $f_{1}, \ldots, f_{r}: \mathbb{F}_{p} \rightarrow \mathbb{C}$ to be

$$
\left(f_{1} * \cdots * f_{r}\right)(n):=\mathbb{E}_{z_{1}, \ldots, z_{r-1}} f_{1}\left(z_{1}\right) \cdots f_{r-1}\left(z_{r-1}\right) f_{r}\left(n-z_{1}-\cdots-z_{r-1}\right) .
$$

Finally, for a function $f: \mathbb{F}_{p} \rightarrow \mathbb{C}$, we define the "support of $f$ ", denoted as

$$
\operatorname{supp}(f) \subseteq \mathbb{F}_{p}
$$

to be the places $a \in \mathbb{F}_{p}$ where $f(a) \neq 0$.

Our main theorem of the paper, from which our results on sumsets $S+S$ follows as an easy consequence, is stated as follows:

THEOREM 1. Let $p$ be a prime number and suppose that the function $f: \mathbb{F}_{p} \rightarrow \mathbb{R}_{\geq 0}$ does not vanish identically. If , for real $\varepsilon$ and positive integer $k \leq\lceil(\log p) / \log 2\rceil$ we have $\lambda_{k+1} \leq \varepsilon \lambda_{k}^{2}$, then

$$
|\operatorname{supp}(f * f)| \geq\left(1-2 \theta \varepsilon^{2}\right) p \text {, where } \theta:=\mathbb{E}\left(f^{2}\right) .
$$

REMARK 1. By letting $f$ be the indicator function for $S$, we see that $\theta=\mathbb{E}\left(f^{2}\right)=\mathbb{E}(f)=|S| / p$, which is the density of $S$ relative to $\mathbb{F}_{p}$. Also, $\operatorname{supp}(f * f)$ is just $S+S$.

REMARK 2. It is easy to construct functions $f$ which have a large spectral gap as in the hypotheses. For example, take $f$ to be the function whose Fourier transform satisfies $\widehat{f}(0)=1 / 2, \widehat{f}(1)=\widehat{f}(-1)=1 / 4$, and $\widehat{f}(a)=0$ for $a \neq 0, \pm 1$. Clearly, we have $f: \mathbb{F}_{p} \rightarrow[0,1]$, and of course $f$ has a large spectral gap between $\lambda_{3}$ and $\lambda_{4}\left(\lambda_{3}=1 / 4\right.$, while $\left.\lambda_{4}=0\right)$.

REMARK 3. An obvious question that one can ask regarding the above theorem is whether it is possible to relax the condition $\lambda_{k+1} \leq \varepsilon \lambda_{k}^{2}$. In 
particular, it would be desirable to reduce the exponent below 2 . This seems to be a difficult problem to address, as it is not even known how to improve the exponent for the case $k=1$, where a large spectral gap corresponds to the assertion that the function $f$ is quasirandom. An example indicating that reducing the exponent near to 1 might be hopeless is given as follows: Suppose that $A$ is a random subset of $\mathbb{F}_{p}$ of size $o(\sqrt{p})$; then $\lambda_{2}=\varepsilon \lambda_{1}$ with $\varepsilon \approx|A|^{-1 / 2}$, while $A+A$ is small as compared to $p$. However, this is not quite a counterexample in the sense that in this case $|A+A|$ is still large compared to $|A|$.

By considering repeated sums, one can prove similar sorts of results, but which hold for a much wider range of $k$. Furthermore, one can derive conditions guaranteeing that $(f * \cdots * f)(n)>0$ for all $n \in \mathbb{F}_{p}$, not just $1-\varepsilon$ proportion of $\mathbb{F}_{p}$. This new theorem is given as follows:

TheOREM 2. Fix $t \geq 3$. Then the following holds for all primes $p$ sufficiently large: Suppose that $f: \mathbb{F}_{p} \rightarrow[0,1], f$ not identically 0 , has the property that for some

$$
1 \leq k<(\log p)^{t-1}(5 t \log \log p)^{-2 t+2},
$$

we have that

$$
\lambda_{k+1}<\lambda_{k}^{t} / t \theta^{t-2}, \text { where } \theta:=\mathbb{E}(f) .
$$

(Note that $\theta$ was defined differently in Theorem 1.) Then the t-fold convolution $f * \cdots * f$ is positive on all of $\mathbb{F}_{p}$.

REMARK. It is possible to sharpen this theorem so that $t$ is allowed to depend on $p$ in some way, though we will not bother to develop this here.

We conjecture that it is possible to prove a lot more:

CONJeCTURE. The logarithmic bound on $k$ in Theorem 1 can be replaced with an exponential bound of the sort $k<p^{c}$ with a constant $c>0$.

This would obviously require a different sort of proof than appears in the present paper.

2. Some lemmas. First, we will require the following standard consequence of Dirichlet's box principle; its proof is also standard, so we will omit it:

Lemma 1. Suppose that

$$
r_{1}, \ldots, r_{t} \in \mathbb{F}_{p} .
$$

Then there exists non-zero $m \in \mathbb{F}_{p}$ such that

$$
\left\|\frac{m r_{i}}{p}\right\| \leq p^{-1 / t} \quad \text { for } i=1, \ldots, t,
$$

where $\|x\|$ denotes the distance from $x$ to the nearest integer. 
The following was first proved by Browkin, Diviš and Schinzel [2] and is also a consequence of much more robust results due to Bilu, Lev and Ruzsa [1] and Lev [5] (unlike [1], this last paper of Lev addresses the case of arbitrary abelian groups) $\left({ }^{2}\right)$.

Lemma 2. Suppose that

$$
B:=\left\{b_{1}, \ldots, b_{t}\right\} \subseteq \mathbb{F}_{p} .
$$

If

$$
t \leq\lceil(\log p) / \log 2\rceil,
$$

then there exists $d \in \mathbb{F}_{p}$ having a unique representation as a difference of two elements of $B$.

Finally, we will also need the following lemma, which is a refinement of one appearing in [6]:

Lemma 3. Suppose that

$$
B_{1}, B_{2} \subseteq \mathbb{F}_{p} \text {, where } 10 \leq\left|B_{1}\right| \leq p / 2 \text { and }\left|B_{1}\right| \geq\left|B_{2}\right| .
$$

$$
2\left|B_{2}\right| \log \left|B_{1}\right|<\log p,
$$

then there exists $d \in B_{1}-B_{2}$ having a unique representation as $d=b_{1}-b_{2}$, $b_{i} \in B_{i}$; on the other hand, if

$$
2\left|B_{2}\right| \log \left|B_{1}\right| \geq \log p,
$$

then there exists $d \in B_{1}-B_{2}$ having at most

$$
20\left|B_{2}\right|\left(\log \left|B_{1}\right|\right)^{2} / \log p
$$

representations as $d=b_{1}-b_{2}, b_{i} \in B_{i}$.

Proof. Suppose that (1) and (2) hold. Then, by Lemma 1, there exists $m$ such that for every $x \in C_{2}:=m \cdot B_{2}$ we have $|x| \leq p /\left|B_{1}\right|^{2}$; furthermore, by the pigeonhole principle there exists an integer interval $I:=(u, v) \cap \mathbb{Z}$ with $u, v \in C_{1}:=m \cdot B_{1}$, with $|I| \geq p /\left|B_{1}\right|-1$, which contains no elements of $B_{1}$. So, $v-\max _{x \in C_{2}} x$ has a unique representation as a difference $c_{1}-c_{2}$, $c_{1} \in C_{1}, c_{2} \in C_{2}$. The same holds for $B_{1}-B_{2}$, and so this part of our lemma is proved.

Now we suppose that (1) and (3) hold. Let $B^{\prime}$ be a random subset of $B_{2}$, where each element $b \in B_{2}$ lies in $B^{\prime}$ with probability

$$
(\log p) /\left(3\left|B_{2}\right| \log \left|B_{1}\right|\right) \text {. }
$$

$\left({ }^{2}\right)$ Straus [7] proved a weaker form of this lemma, which had the upper bound $|B| \leq$ $(\log p) / \log 4$ in place of $|B| \leq\lceil(\log p) / \log 2\rceil$. He remarked that Feit had first brought the problem to his attention. The first-named author of the present paper rediscovered a proof of this result, as appeared in an earlier version of the text. Recently, Jańczak [3] has proved some extensions of Straus' results to linear combinations of elements of a set $B$. 
Note that this is where our lower bound $2\left|B_{2}\right| \log \left|B_{1}\right| \geq \log p$ comes in, as we need this probability to be at most 1 .

So long as the $B^{\prime}$ we choose satisfies

$$
\left|B^{\prime}\right|<(\log p) /\left(2 \log \left|B_{1}\right|\right),
$$

which it will with probability at least $1 / 3$ by an easy application of Markov's inequality, we claim that there will always exist an element $d \in B-B^{\prime}$ having a unique representation as a difference $b_{1}-b_{2}^{\prime}, b_{1} \in B, b_{2}^{\prime} \in B^{\prime}$ : First, note that it suffices to prove this for the set $C_{1}-C^{\prime}$, where

$$
C_{1}=m \cdot B_{1}, \quad C_{2}=m \cdot B_{2}, \quad C^{\prime}=m \cdot B^{\prime},
$$

and where $m$ is a dilation constant chosen according to Lemma 1 , so that every element $x \in C^{\prime}$ (when considered as a subset of $(-p / 2, p / 2]$ ) satisfies

$$
|x| \leq p^{1-1 /\left|B^{\prime}\right|}<p /\left(3\left|B_{1}\right|\right) .
$$

Now, there must exist an integer interval

$$
I:=(u, v) \cap \mathbb{Z}, \quad u, v \in C_{1},
$$

(which we consider as an interval modulo $p$ ) such that

$$
|I| \geq p /\left|C_{1}\right|-1=p /\left|B_{1}\right|-1,
$$

and such that no element of $C_{1}$ is congruent modulo $p$ to an element of $I$. Clearly, then, $v-\max _{c^{\prime} \in C^{\prime}} c^{\prime}$ has a unique representation as a difference.

Now we define the functions

$$
\begin{aligned}
\nu(x) & :=\left|\left\{\left(c_{1}, c_{2}\right) \in C_{1} \times C_{2}: c_{1}-c_{2}=x\right\}\right|, \\
\nu^{\prime}(x) & :=\left|\left\{\left(c_{1}, c_{2}^{\prime}\right) \in C_{1} \times C^{\prime}: c_{1}-c_{2}^{\prime}=x\right\}\right| .
\end{aligned}
$$

We claim that with probability exceeding $2 / 3$,

(5) every $x \in \mathbb{F}_{p}$ with $\nu(x)>20\left|B_{2}\right|\left(\log \left|B_{1}\right|\right)^{2} / \log p$ satisfies $\nu^{\prime}(x) \geq 2$.

Note that since the sum of $\nu(x)$ over all $x \in \mathbb{F}_{p}$ is $\left|B_{1}\right| \cdot\left|B_{2}\right|$, the number of $x$ satisfying this hypothesis on $\nu(x)$ is at most, for $p$ sufficiently large,

$$
\frac{\left|B_{1}\right| \cdot\left|B_{2}\right|}{20\left|B_{2}\right|\left(\log \left|B_{1}\right|\right)^{2} / \log p}=\frac{\left|B_{1}\right| \log p}{20\left(\log \left|B_{1}\right|\right)^{2}}<\left|B_{1}\right|,
$$

by (3) and the fact that $\left|B_{1}\right| \geq\left|B_{2}\right|$.

To see that (5) holds, fix $x \in C_{1}-C_{2}$. Then, $\nu^{\prime}(x)$ is the following sum of independent Bernoulli random variables:

$$
\nu^{\prime}(x)=\sum_{j=1}^{\nu(x)} X_{j}, \quad \text { where } \operatorname{Prob}\left(X_{j}=1\right)=(\log p) /\left(3\left|B_{2}\right| \log \left|B_{1}\right|\right) .
$$

The variance of $\nu^{\prime}(x)$ is

$$
\sigma^{2}=\nu(x) \operatorname{Var}\left(X_{1}\right) \leq \nu(x) \mathbb{E}\left(X_{1}\right) .
$$


We will now need the following well-known theorem of Chernoff:

Theorem 3 (Chernoff's inequality). Suppose that $Z_{1}, \ldots, Z_{n}$ are independent random variables such that $\mathbb{E}\left(Z_{i}\right)=0$ and $\left|Z_{i}\right| \leq 1$ for all $i$. Let $Z:=\sum_{i} Z_{i}$, and let $\sigma^{2}$ be the variance of $Z$. Then

$$
\operatorname{Prob}(|Z| \geq \delta \sigma) \leq 2 e^{-\delta^{2} / 4} \quad \text { for any } 0 \leq \delta \leq 2 \sigma .
$$

We apply this theorem using $Z_{i}=X_{i}-\mathbb{E}\left(X_{i}\right)$ and

$$
\delta \sigma=\nu(x) \mathbb{E}\left(X_{1}\right)-1,
$$

and then deduce that if $\nu(x)>20\left|B_{2}\right|\left(\log \left|B_{1}\right|\right)^{2} / \log p$, then

$$
\operatorname{Prob}\left(\nu^{\prime}(x) \leq 1\right)=\operatorname{Prob}\left(Z \leq 1-\nu(x) \mathbb{E}\left(Z_{1}\right)\right) .
$$

Noting that $1-\nu(x) \mathbb{E}\left(Z_{1}\right)<0$, we deduce that

$$
\operatorname{Prob}(|Z| \leq \delta \sigma) \leq 2 \exp \left(-\delta^{2} / 4\right) \leq 2 \exp \left(-\frac{\left(\nu(x) \mathbb{E}\left(X_{1}\right)-1\right)^{2}}{4 \nu(x) \mathbb{E}\left(X_{1}\right)}\right)<\frac{1}{3\left|B_{1}\right|}
$$

Clearly, since there are at most (6) places $x$ where $\nu(x)$ satisfies the hypotheses of (5), it follows that (5) holds with probability exceeding $2 / 3$. But also (4) holds with probability at least $1 / 3$; so, there is an instantiation of the set $B^{\prime}$ such that both (5) and (4) hold. Since we proved that such a $B^{\prime}$ has the property that there is an element $x \in B_{1}-B^{\prime}$ having $\nu^{\prime}(x)=1$, it follows from (5) that $\nu(x) \leq 20\left|B_{2}\right|\left(\log \left|B_{1}\right|\right)^{2} / \log p$, which proves the second part of our lemma.

3. Proof of Theorem 1. We apply Lemma 2 with $B=A=\left\{a_{1}, \ldots, a_{k}\right\}$, so $t=k$. Let then $d$ be as in the lemma, and let $a_{x}, a_{y} \in A$ satisfy

$$
a_{y}-a_{x}=d .
$$

We define

$$
g(n):=e^{2 \pi i d n / p} f(n),
$$

and note that

$$
(f * f)(n) \geq|(g * f)(n)| .
$$

So, our theorem is proved if we can show that $(g * f)(n)$ is often non-zero. Proceeding in this vein, let us compute the Fourier transform of $g * f$ : First, we have

$$
\widehat{g}(a)=\mathbb{E}_{n}\left(g(n) e^{2 \pi i a n / p}\right)=\mathbb{E}_{n}\left(f(n) e^{2 \pi i n(a+d) / p}\right)=\widehat{f}(a+d) .
$$

So, by Fourier inversion,

$$
(f * g)(n)=e^{-2 \pi i a_{x} n / p} \widehat{f}\left(a_{x}\right) \widehat{f}\left(a_{y}\right)+E(n),
$$

where $E(n)$ is the "error" given by

$$
E(n)=\sum_{a \neq a_{x}} e^{-2 \pi i a n / p} \widehat{f}(a) \widehat{f}(a+d) .
$$


Note that for every value of $a \neq a_{x}$ we have

(8) either $a$ or $a+d$ lies in $\left\{a_{k+1}, \ldots, a_{p}\right\}$

$$
\Rightarrow|\widehat{f}(a) \widehat{f}(a+d)| \leq \varepsilon \lambda_{k}^{2} \max \{|\widehat{f}(a)|,|\widehat{f}(a+d)|\} .
$$

To finish our proof we must show that "most of the time" $|E(n)|$ is smaller than the "main term" of (7); that is,

$$
|E(n)|<\left|\widehat{f}\left(a_{x}\right) \widehat{f}\left(a_{y}\right)\right| .
$$

Note that this holds whenever

$$
|E(n)|<\lambda_{k}^{2} \text {. }
$$

We deduce by Parseval and (8) that

$$
\begin{aligned}
\sum_{n}|E(n)|^{2} & =p \sum_{a \neq a_{x}}|\widehat{f}(a)|^{2}|\widehat{f}(a+d)|^{2} \leq 2 p \varepsilon^{2} \lambda_{k}^{4} \sum_{a}|\widehat{f}(a)|^{2} \\
& \leq 2 p \varepsilon^{2} \lambda_{k}^{4} \mathbb{E}\left(f^{2}\right)=2 p \varepsilon^{2} \lambda_{k}^{4} \theta .
\end{aligned}
$$

So, the number of $n$ for which (9) holds is at least $p\left(1-2 \theta \varepsilon^{2}\right)$, as claimed.

\section{Proof of Theorem 2. Let}

$$
B_{1}:=B_{2}:=A=\left\{a_{1}, \ldots, a_{k}\right\} .
$$

Suppose initially that $2|A| \log |A| \geq \log p$, so that the hypotheses of the second part of Lemma 3 hold. We then see that there exists $d_{1} \in B_{1}-B_{2}=$ $A-A$ with at most $20|A|(\log |A|)^{2} / \log p$ representations as $d_{1}=a-b$, $a, b \in A$. Let now $A_{1}$ denote the set of all the elements $b$ that occur. Clearly,

$$
\left|A_{1}\right| \leq 20|A|(\log |A|)^{2} / \log p .
$$

Keeping $B_{1}=A$, we reassign $B_{2}=A_{1}$. So long as $2\left|A_{1}\right| \log |A| \geq \log p$ we may apply the second part of Lemma 3 , and when we do we deduce that there exists $d_{2} \in A-A_{1}$ having at most $20\left|A_{1}\right|(\log |A|)^{2} / \log p$ representations as $d_{2}=a-b, a \in A, b \in A_{1}$. Let now $A_{2}$ denote the set of all elements $b$ that occur. Clearly,

$$
\left|A_{2}\right| \leq 20\left|A_{1}\right|(\log |A|)^{2} / \log p .
$$

We repeat this process, reassigning $B_{2}=A_{2}$, then $B_{2}=A_{3}$, and so on, all the while producing these sets $A_{1}, A_{2}, \ldots$, and differences $d_{1}, d_{2}, \ldots$, until we reach a set $A_{m}$ satisfying

$$
2\left|A_{m}\right| \log |A|<\log p .
$$

We may, in fact, reach this set $A_{m}$ with $m=1$ if $2|A| \log |A|<\log p$ to begin with.

It is clear that since at each step we have, for $i \geq 2$,

$$
\left|A_{i}\right| \leq 20\left|A_{i-1}\right|(\log |A|)^{2} / \log p<\left|A_{i-1}\right|(5 \log |A|)^{2} / \log p
$$


it follows that

$$
\left|A_{i}\right| \leq|A|(5 \log |A|)^{2 i} /(\log p)^{i} .
$$

Since we have assumed that

$$
|A|<(\log p)^{t-1}(5 t \log \log p)^{-2 t+2},
$$

were we to continue our iteration to $i=t-1$ we would have

$$
\left|A_{t-1}\right|<|A|(5 \log |A|)^{2 t-2} /(\log p)^{t-1}<(t \log \log p)^{-2 t+2}(\log |A|)^{2 t-2} \ll_{t} 1 .
$$

So, our number of iterations $m$ satisfies

$$
m \leq t-1,
$$

for $p$ sufficiently large.

By the second part of Lemma 3 , this set $A_{m}$ will have the property that there exists $d_{m} \in A-A_{m}$ having a unique representation as $d_{m}=a-b$, $a \in A, b \in A_{m}$.

Now, we claim that there exists a unique $b \in \mathbb{F}_{p}$ such that

$$
b, b+d_{1}, b+d_{2}, \ldots, b+d_{m} \in A .
$$

To see this, first let $b \in A$. Since $b+d_{1} \in A$ we must have $b \in A_{1}$, by definition of $A_{1}$. Then, since $b+d_{2} \in A$, it follows that $b \in A_{2}$. And, repeating this process, we eventually conclude that $b \in A_{m}$.

So, since $b \in A_{m}$, and $b+d_{m} \in A$, we have $d_{m}=a-b, a \in A, b \in A_{m}$. But this $d_{m}$ was chosen by the second part of Lemma 3 so that it has a unique representation of this form. It follows that $b \in A$ is unique, as claimed.

From our function $f: \mathbb{F}_{p} \rightarrow[0,1]$, we define the functions $g_{1}, \ldots, g_{m}$ : $\mathbb{F}_{p} \rightarrow \mathbb{C}$ via

$$
f_{i}(n):=e^{2 \pi i d_{i} n / p} f(n) .
$$

It is obvious that

$$
\operatorname{supp}\left(f * \cdots * f * g_{1} * \cdots * g_{m}\right) \subseteq \operatorname{supp}(f * \cdots * f),
$$

where there are $t$ convolutions on the left, and $t$ on the right; so, $f$ appears $t-m$ times on the left.

We also have

$$
\widehat{g}_{i}(a)=\widehat{f}\left(a+d_{i}\right),
$$

and therefore

$$
\left(f * \cdots * f * g_{1} * \cdots * g_{m}\right)^{\wedge}(a)=\widehat{f}(a)^{t-m} \widehat{f}\left(a+d_{1}\right) \cdots \widehat{f}\left(a+d_{m}\right) .
$$

Since there exists a unique $a$, call it $x$, such that all these $a+d_{i}$ belong to $A$, we deduce via Fourier inversion that for any $n \in \mathbb{F}_{p}$,

$$
\left(f * \cdots * f * g_{1} * \cdots * g_{m}\right)(n)=e^{-2 \pi i n x / p} \widehat{f}(x)^{t-m} \widehat{f}\left(x+d_{1}\right) \cdots \widehat{f}\left(x+d_{m}\right)+E(n),
$$


where the "error" $E(n)$ satisfies, by the usual $L^{2}-L^{\infty}$ bound,

$$
|E(n)| \leq t \lambda_{k+1} \theta^{t-3} \sum_{a}|\widehat{f}(a)|^{2}<\lambda_{k}^{t} .
$$

So, since all of $|\widehat{f}(a)|,\left|\widehat{f}\left(a+d_{1}\right)\right|, \ldots,\left|\widehat{f}\left(a+d_{m}\right)\right|$ are bounded from above by $\lambda_{k}$, we find that $|E(n)|$ is smaller than our main term above, and therefore $(f * \cdots * f)(n)>0$.

Acknowledgements. We would like to thank Vsevolod Lev for the numerous helpful comments and suggestions, and to thank Liangpan Li for pointing out the reference [3].

Research of E. Croot supported in part by an NSF grant. Research of T. Schoen was partially supported by MNSW grant 2 P03A 02930.

\section{References}

[1] Y. F. Bilu, V. F. Lev and I. Z. Ruzsa, Rectification principles in additive number theory, Discrete Comput. Geom. 19 (1998), 343-353.

[2] J. Browkin, B. Diviš and A. Schinzel, Addition of sequences in general fields, Monatsh. Math. 82 (1976), 261-268.

[3] M. Jańczak, A note on a problem of Hilliker and Straus, Electron. J. Combin. 14 (2007), Note 23.

[4] V. F. Lev, Simultaneous approximations and covering by arithmetic progressions in $\mathbb{F}_{p}$, J. Combin. Theory Ser. A 92 (2000), 103-118.

[5] - Rectifiability threshold in abelian groups, Combinatorica, to appear.

[6] T. Łuczak and T. Schoen, On a problem of Konyagin, Acta Arith. 134 (2008), 101109.

[7] E. G. Straus, Differences of residues $(\bmod p)$, J. Number Theory 8 (1976), 40-42.

School of Mathematics

Georgia Tech

103 Skiles

Atlanta, GA 30332, U.S.A.

E-mail: ecroot@math.gatech.edu
Department of Discrete Mathematics

Adam Mickiewicz University

Umultowska 87

61-614 Poznań, Poland

E-mail: schoen@amu.edu.pl

Received on 7.2.2008

and in revised form on 13.8.2008 